\title{
Comparison of process documentation generation in PTC Creo and NX systems
}

\author{
Martin Pollák ${ }^{1, *}$, Petr Baron ${ }^{1}$, and Marek Kočiško ${ }^{1}$ \\ ${ }^{1}$ Technical University of Košice, Faculty of Manufacturing Technologies, 08001 Prešov, Slovakia
}

\begin{abstract}
Process documentation is a specific type of progressive form for representation of the technological documentation which defines the sequence of the individual production steps on the chosen machine according to a pre-executed NC program. It is represented by a simplified form of automatically generated reports in a short time interval. The significant contribution of the creation is in the acceleration of communication between the designer and the technologist, as well as $\mathrm{NC}$ machine operators with the possibility of archiving and managing internal data. The article briefly describes and compares the creation of process documentation in the PTC Creo system and in the NX system environment.
\end{abstract}

\section{Introduction}

From the analysis of the total labour consumption accounts for $70 \%$ to $80 \%$ for the technological preparation of production. Thus, it can be said that this is the most time consuming stage in the production process. Requirements for creating technical documentation need to have a high level of technological preparation. The requirements make sure that the parts are manufactured correctly and the manufacturing steps in sequence. Technological preparation of production uses intuition, logical activity and the routine work mostly. Currently, due to the differences in the creation of technological documentation, it is necessary to divide the technological preparation of the production according to the area of its use and form [1].

Technological documentation is represented in form of [2]:

- technological process written verbally,

- technological process represented by images,

- NC code of program,

- simulation (visual presentation of the technological documentation).

The area of NC machining is a specific area in the production of technological processes. The main document for NC machining is the NC program. The NC program defines the sequence of the individual steps that are executed on the specified machine. Despite the amount of information that the NC program contains, it cannot be said that there is sufficient technology documentation.

The NC program consists of the basic features of the technological process, i.e. it specifies the basic machine setting and defines the precise tool paths as well as some

\footnotetext{
*Corresponding author: martin.pollak@tuke.sk
} 
auxiliary operations (tool change, lubrication, cooling...) [3]. The machine operator is missing additional data (base unit, number of pieces produced, material of the product and the like.)

For this reason, it is necessary to extend this documentation to its other forms. Today's $\mathrm{CAD} / \mathrm{CAM} / \mathrm{CAE}$ systems used to create NC programs offer us three solutions [1]:

- additional use of CAPP systems,

- implementation of complex PLM systems into the manufacturing company,

- possibility to use the automated generation of process documentation in the $\mathrm{CAD} / \mathrm{CAM} / \mathrm{CAE}$ system.

The article points to the creation of process documentation in the PTC Creo 3.0 system and in the NX system environment. The aim of creation is the elimination of lengthy processes during the step of technological preparation of production, using a simple and fast generation of process documentation directly in that system.

\section{Creation of technological documentation and its automation}

Mechanical work in the 20th century began to replace automated technical preparation of production, in order to save time and human forces. Manufacturing and machining equipment began to be produced, which were later replaced by $\mathrm{NC}$ machines. The most valuable aspect of today is the time which is the main reason why new technologies are introduced in the field of development and production. The main task of introducing new technologies into the manufacturing sphere is to shorten the design, production and other processes times to the minimum. The companies gradually began to use and implement a comprehensive CAD/CAM/CAE, CAPP and PLM systems.

The technical preparation of production includes all the activities that need to be carried out before the actual production of the product begins. All these activities are focused on the preparation and processing of product technical documentation (design, technology and projection). Variants of these solutions ensure maximum productivity and efficiency of production processes, such as shorter lead times, material savings, energy savings, labour intensity and production costs [4].

The manufacturing process provides the necessary manufacturing information, such as the times of individual manufacturing steps and intermediate steps, machines and tools used for the individual operations, movement of the tools, cutting speed, etc.

The information contained in the technological process can be divided into:

- the identification data: number of technological process, drawing number,

- the material information: designation of material quality, consumption amount of material per component, total amount of consumed material, initial state of the material,

- the operational data: number of operations, marking of the work, marking of the workplace, type of machine, tools, jigs, gauges, brief description of the operation, number of operational instruction cards, batch time, unit time, tariff class, total time on benefit, total cost of the operation,

- the production data: number of pieces in production batch, marking of the center designated for the production of parts, number of production orders, number of material warehouse.

\section{The process of generating the process documentation}

In the NC machining a technological documentation is based on the principle of a simplified process flow in the form of process documentation. There are already a lot of 
complex $\mathrm{CAD} / \mathrm{CAM} / \mathrm{CAE}$ systems that offer the possibility to use module for the automated generation of process documentation.

The process documentation represents a progressive form of automated generation of simplified technical documentation and is primarily intended for NC machining area. This option resulted from the requirements of the accelerated creation of technological documentation for the deployment of comprehensive CAD/CAM/CAE systems.

\subsection{Generating process documentation in the PTC Creo 3.0 system}

The PTC Creo 3.0 has this option available under the tab Application/Process Documentation. Automated generation is suitable for fast creation of workshop documentation, and its great advantage is the ability to archive in digital form HTML. Generated documentation is therefore possible, at any moment, to be customized, defined and used as a simplified form of representation of technological process. The Creo 3.0 provides two options for the creation of process documentation using predefined templates by:

- Process Documentation Defaults,

- Process Manager Defaults.

By selecting a template generation via Process Document Defaults are looking at a Report by operation choice of two types of display as follows: Process Main View and Tool View. The first type Process Main View contains a list of operation names and types of operations. In the second type, the Tool View is locating a system-preset list of tools and items such as:

- Name (name of operation),

- Tool_ID (tool name),

- Tool_Type,

- Cutter_Diam,

- Tool_Comment.

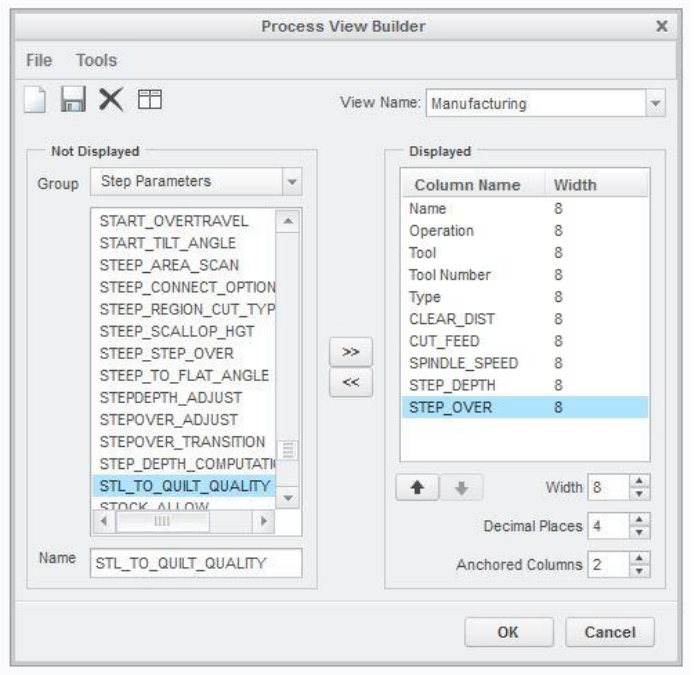

Fig. 1. Process View Builder.

Documentation generation content can be changed in accordance with the necessary data for production. The auxiliary window Process View Builder (Fig. 1) is divided into two parts that are not displayed and Displayed (not shown and displayed items) which determines the content of process documentation data. By gradually adding of items using 
the tool inserted, the user sets the appearance of process documentation. The selected data can then be sorted in the selected order of Displayed. In order to avoid of repeated and lengthy selection, the list of items can be saved separately.

After editing the appearance of the template settings are saved. Before the start of generation it is necessary to be able to select the desired look of generated process documentation. Process documentation may have a look as simple text or can be supplemented by graphic illustrations of operations and tools.

After checking a field Manufacturing Model Image, Step Image, eventually Tool and Solid Tool Image the choice in the generated documentation process reflected in the representations of the graphic representation of the machined areas, and also a graphic illustration of the use of tools. By the icon Create is launches the generation process (Fig. 2).

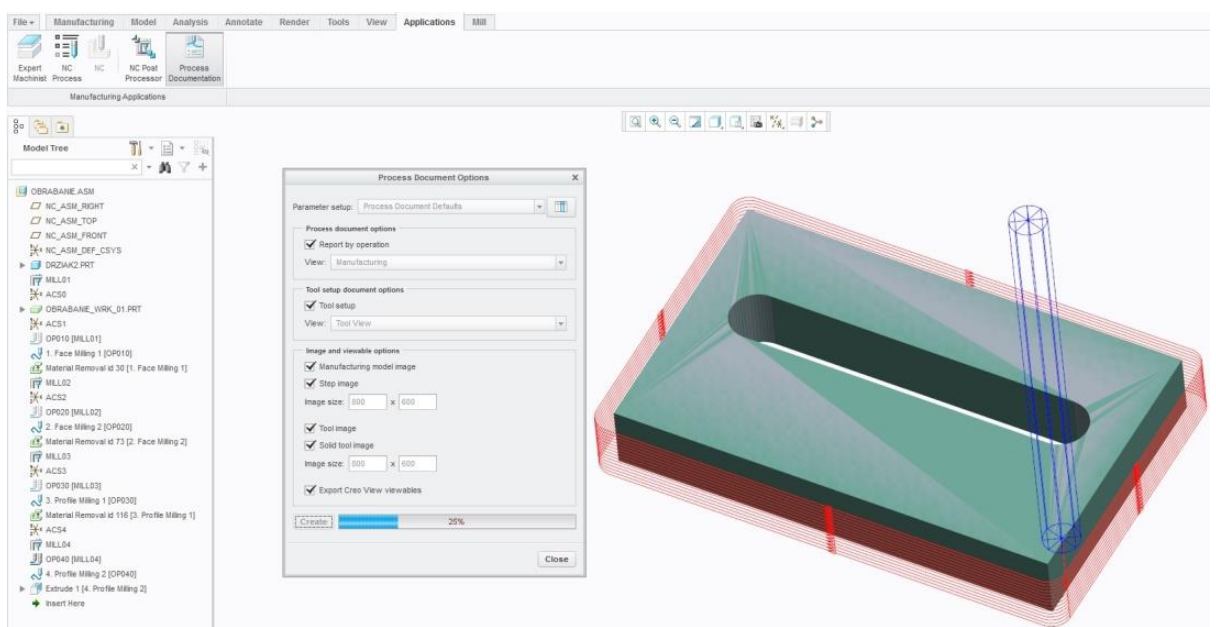

Fig. 2. The process of generating the process documentation.

After finishing the video demonstration, the system generates process documentation in HTML format (Fig. 3).

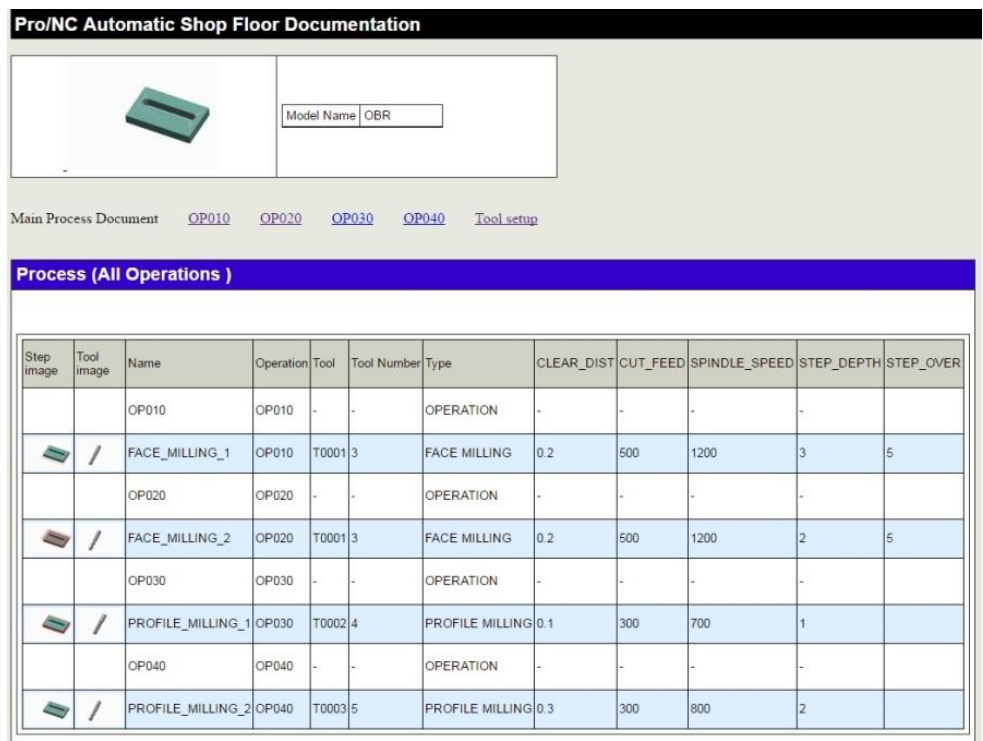

Fig. 3. Process documentation generated in the PTC Creo 3.0 system. 
By choosing of determined sequence of machining through graphical interpretation, the system displays more detailed magnified picture presenting view of the machining areas with exact specifications of set machining operation as well as information about the tool with which a given area is machined.

In the system PTC Creo 3.0 it is not possible in the generation of process documentation to change the look or format of documentation, which is the only disadvantage in the generating process. However, generated values in the table can be changed.

\subsection{Generating process documentation in the NX system}

Process documentation (in the NX system called "Shop Documentation") can be formed by four ways to view the machine sequences in terms of program, tool geometry and machining methods. These data can be viewed in a generated documentation using a set of objects that offers individual display options (machining sequences, geometry and component material, machining parameters, tool parameters, tool path information, tool parameter controls, control parameters...).

In the NX system, it is possible to create shop documentation using 4 predefined templates:

- Operation List Select (HTML/Excel) - generating a template in terms of machining operations in HTML format with editable templates in MS Excel (Fig. 4).

- Operation List Select (ASCII) - generating a template in terms of machining operations in the ASCII text format with the possibility of printing and distributing reports.

- Tool List Select (HTML/Excel) - generating a template in terms of used cutting tools with defined machining parameters in HTML with the ability to edit the template in the MS Excel editor. Tools are assigned to individual machining sequences. The tools are then, in the complete tool list, ordered by their assigned name.

- Tool List Select (ASCII) - generating a template in terms of used cutting tools with defined machining parameters in text format (without graphic elements and hyperlinks).

HTML (web) format presents data that can be browsed through a web browser and the ability to share documents on the web. In the created shop documentation, the graphs of the individual designs are displayed in the form of images with clear browsing through hyperlinks.

Program Sheet
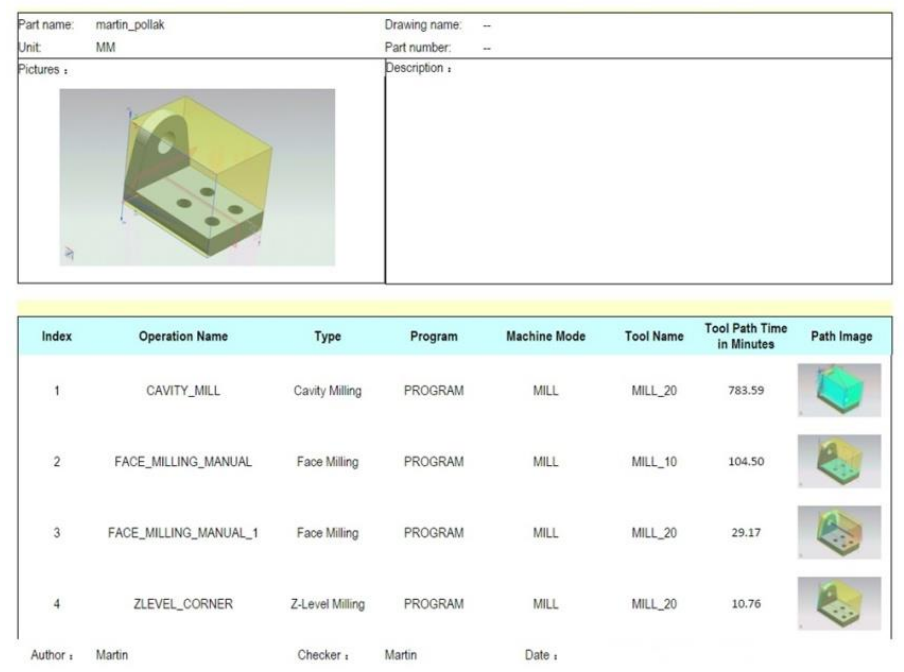

Fig. 4. Shop documentation generated in terms of machining operations in HTML format. 
The template created in HTML format can be edited in the Microsoft Excel spreadsheet environment. Editing is done in the MS Excel worksheet file named shopdoc_template.xlsx (Fig. 5). Editing the file creates a new layout template according to the user's needs. Excel template file consists of two worksheets. The first worksheet includes a visual template page with the option of editing the layout, cell dimensions, data entry sequences and other editing options.

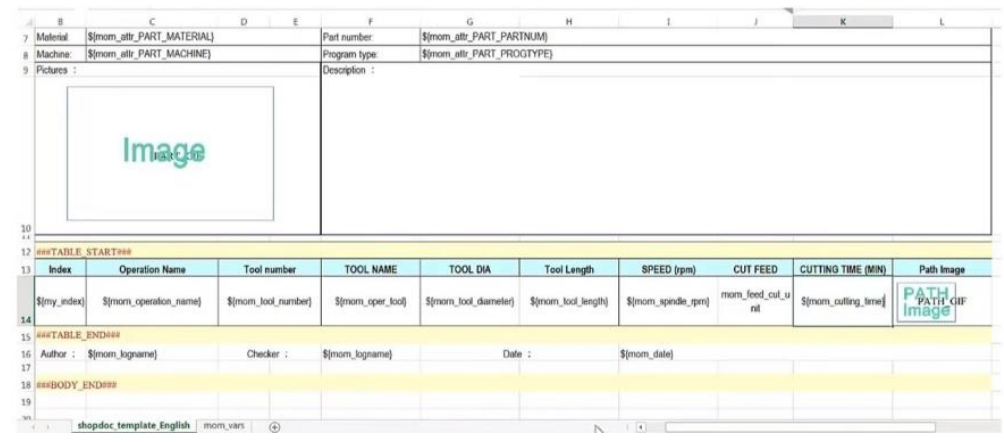

Fig. 5. Modification of shop documentation in MS Excel environment.

The second Excel worksheet contains an overview and description of the variables that are needed to produce changes in the first Excel worksheet, by simply copying and pasting the variables in the template cells (MOM). Variables are written to facilitate creation, without the need to study the linguistic expression statements of MOM variables.

\section{Conclusion}

Creation of process documentation is currently a required part of complex CAD/CAM/CAE systems. Therefore, the developers of these systems are continuously developing and incorporating this creation option into their products. Generating process documentation is relatively new and underdeveloped tool despite the efforts of the creators. Process documentation is simplified in the form of automatically generated reports containing the necessary parameters and graphical interpretation of specific machining cycles created in the system CAM environment of the given system.

The most important benefits of using automated generating of process documentation are:

- process documentation is a comprehensive, organized form of data that simplifies communication between the constructor, technology and NC machine operators,

- speed of processing of larger amount of information into a single document with the absence of the manual writing of technological documentation in the additional CAPP systems,

- outputs from these modules can also be used to archive and manage internal records, making it easier for management to produce productivity analyses,

- it allows quick upload of process documentation on the internet, because the output is directly in HTML, which improves communication between employees in different departments,

- process documentation can be used in training of new operators by providing information on the current project.

Each software maker has chosen a different approach to creating these templates. It should be noted that this option is not intended for normal system users. The user's simplest way to define a custom template is provided by PTC Creo but, on the other hand, the possibility of change is limited only to changing the individual displayed parameters in the 
output documentation (graphical form and visual appearance of documentation cannot be changed). The NX system provides wider possibilities for defining templates but there is a need to have knowledge of programming, technology and detailed knowledge of the system at the administrator's level. This system allows the creation of any graphics format of final documentation, which is a great advantage compared to the system PTC Creo 3.0.

The Ministry of Education, Science, Research and Sport of SR supported this work, contract VEGA No. 1/0492/16, and ITMS project 26220220125.

\section{References}

1. M. Staniszewski, S. Legutko, P. Raos, Tehnicki vjesnik-technical gazette, 21, 11771181, ISSN 1330-3651 (2014)

2. I. Kuric, J. Kosturiak, A. Janac, J. Peterka, J. N. Marcincin, Computer aided systems in mechanical engineering, EDIS, 351, ISBN:80-7100-948-2 (2002)

3. P. Monka, K. Monkova, M. Balara, S. Hloch, J. Rehor, A. Andrej, M. Somsak, International journal of advanced manufacturing technology, 85, 2325-2343, ISSN: 0268-3768 (2016)

4. S. Olejarova, J. Dobransky, J. Svetlik, M. Pituk, Measurement, 106, 18-25, ISSN: 0263-2241 (2017) 
